\title{
Development of a prototype for remote current measurements of PV panel using WSN
}

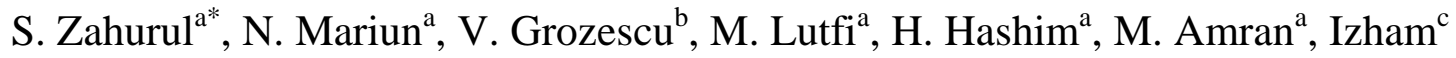 \\ ${ }^{a}$ Dept of Elec Eng, Universiti Putra Malaysia, Serdang 43400, Malaysia \\ ${ }^{b}$ Faculty of Science, Universiti Putra Malaysia, Serdang 43400, Malaysia \\ ${ }^{c}$ Electrical Power Engineering, Kajang 43000, Universiti Tenaga Nasional, Malaysia
}

\begin{abstract}
Sensing, monitoring, actuating and information retrieving are expected to play a key role in smart grid energy management strategy. For energy consumption metering, power measurement systems generally implement transformers contactless current sensors but also have a wide variety of technologies especially for integrating renewable generations. One of the key problems of future smart grid is to develop data communication system for distributed intermittent renewable generations to build an efficient energy management and demand response system. In this paper, a test bed has been developed using Wireless Sensor Network (WSN) based on IEEE 802.15.4 standard for remote real time monitoring of current production in a distributed Photovoltaic (PV) plant. ZigBee based WSN is integrated with Arduino microcontroller and current sensor to sense produce current by PV at every moment and forward this data to control unit instantaneously. In the control unit, a LabVIEW based program is developed to receive the data and store in to a database for further processing of energy management by the control unit.
\end{abstract}

Keywords: Hall effect sensor, photovoltaic, ZigBee communication

\section{Introduction}

Having established the motivation to develop sensor technology in power industry or in other word smart grid communication technology, it is now necessary to come up with a genuine innovation. Traditional current sensors are applied to measure the current in household and industrial sectors, but the measured data is particularly retrieved by visiting manpower. However, once meter data is available in electronic form, it becomes feasible to add communications to the meter, allowing the meter to use Automatic Meter Reading (AMR) to access data remotely via the communication link. Meter manufacturers have developed different system architectures for remote reading, broadly classified as walk-by, drive-by, or networked systems [1]-[5].

Among all the Wireless communication technologies, IEEE 802.15.4 standard ZigBee has a great implication in smart grid communication especially in Advanced Metering Infrastructure (AMI) [6]-[8]. For instance, ZigBee supported features in smart energy profile are demand response and load control, dynamic pricing, text messages, device support for programmable communicating thermostats, load controllers, energy management systems, in-home-displays etc. The profiles are not limited to energy, but also can be implemented in gas and water sectors. Fig. 1 shows the application of ZigBee in smart home management system.

ZigBee technology is created on the IEEE 802.15.4 standard. IEEE 802.15.4 is considered as economic, high-efficiency, low-rate standard for peer-to-peer communication networks. This standard supports band such as $2.4 \mathrm{GHz}$ worldwide, $868 \mathrm{MHz}$ (Europe) and 928MHz (North America) with twoway wireless data transmission. IEEE 802.15.4 standard recognizes physical layer and media access layer (MAC). On this basis, ZigBee Alliance expands the structure of the network layer and application layer.

\footnotetext{
* Manuscript received June 15, 2013; revised August 20, 2013.

Corresponding author. Tel.: +60-193003786; E-mail address: zahurul2003_du@yahoo.com; norman@eng.upm.edu.my.
} 
Therefore, it does not recognize any Internet Protocol (IP) base application. ZigBee is a kind of micropower wireless communication technology and the characters of some typical micro-power wireless communication technologies. The comparison scenario among these communication standards are listed in Table $1[10]$.

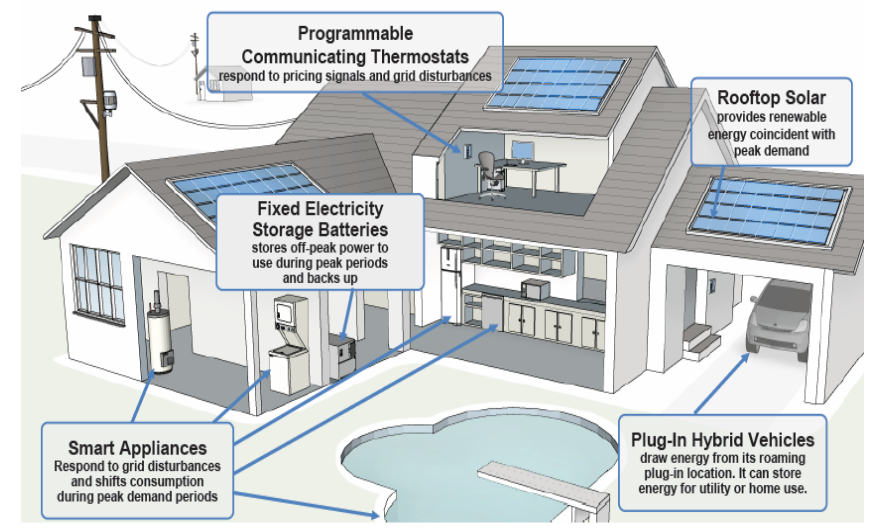

Fig. 1. Application of Zigbee smart energy [9].

Table 1. Typical micro-power wireless communication technologies [10]

\begin{tabular}{llll}
\hline \hline Standard & 802.15.1, Bluetooth & $\mathbf{8 0 2 . 1 1 b}$, WiFi & 802.15.4, ZigBee \\
\hline Application area & Wire substitute & Wireless access & Monitoring and control \\
Bandwidth / (kbps) & $1000 \sim 3000$ & $11000+$ & $20 \sim 250$ \\
Number of nodes & 7 & 32 & 65,000 \\
Technical advantages & Cost, Efficiency & Bandwidth, Flexibility & Power consumption, Cost \\
\hline \hline
\end{tabular}

To date, wide range monitoring and analysis of distributed renewable energy sources does not yet exist, especially in Malaysia. Implementing a supervision and diagnostic system suitable for distribution renewable energy source is beneficial to real-time monitoring that provide information peak load control and demand response. Locations of the distribution renewable sources (off-grid and grid-connected) can be in various places based on grids plans. Therefore, current measurement sensor integrated with ZigBee wireless sensor network (WSN) can be suitable to deploy in distributed renewable sources to achieve ubiquitous real-time electric current production, temperature, and solar irradiance variation data due to environmental factors.

In this paper, first we have figured out the common electricity measurement sensors (or current sensors) that are widely used in electrical meters to measure the electricity consumption. Then, we have discussed about development of a test bed using IEEE 802.15.4 based Wireless Sensor Network for remote current measurement of PV generation. Moreover, a LabVIEW program is integrated with ZigBee serial communication to receive and analyze the produced current using.

In Section 2, presents the methodology of integration ZigBee and Arduino with current sensors elaborately. A technique for retrieving data from ZigBee using LabVIEW program is discussed in Section 3. This technique includes the programming architecture with flow chart diagram. Finally, conclusion is presented in Section 4.

\section{Current Sensors Integrated With ZigBee and Arduino}

Arduino is a tool for making computers that can sense and control more of the physical world than desktop computer. It is an open-source physical computing platform based on a microcontroller board, and a development environment for writing program for the board. Arduino can be used to in various perspectives, however integrated of current sensor is one of the interactive jobs to measure current for different applications. The objective of using Arduino is to make it stand-alone; therefore, it can be placed 
at distributed location. The program installed in Arduino can communicate and send measure current with a computer through Zigbee. Fig. 2 shows the block diagram of communication scenario of the project.

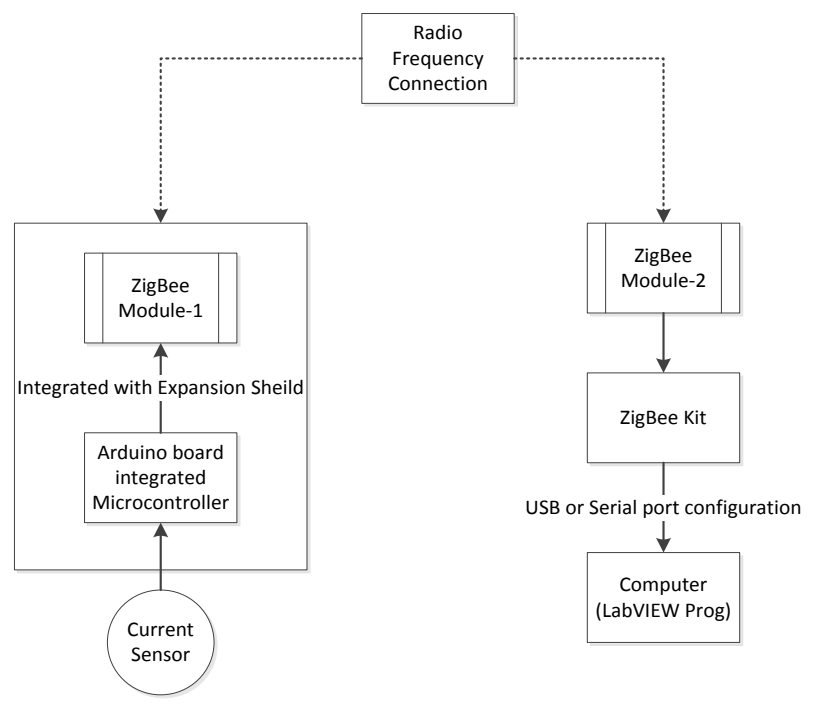

Fig. 2. Schematic diagram of project.

\subsection{Hardware integration}

Accroding to Fig. 2, the communication is established in two different sides. At the distributed side, Arduino integrated with microcontroller and ZigBee with I/O expansion sheild perform operation in two ways- sense generated current and send it to control center. On the other hand, at the control center side, Arduino main board is connected with computer to receive the data (generated current) that is being sent from distributed side. At this side, a LabVIEW based program is designed to read and analyze data in an interval. Fig. 3 displays the hardware accessories for distributed side and control center respectively.

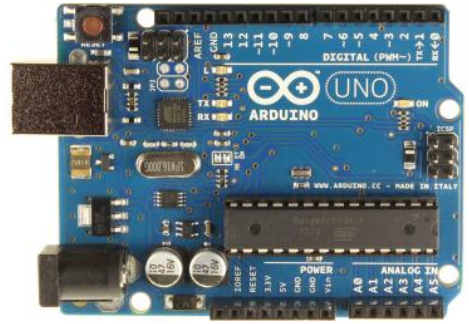

(a)

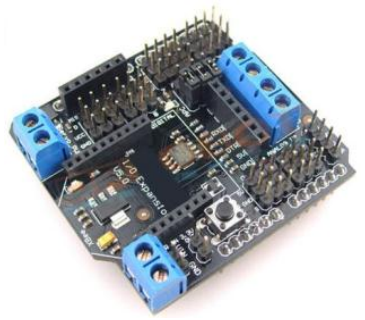

(b)

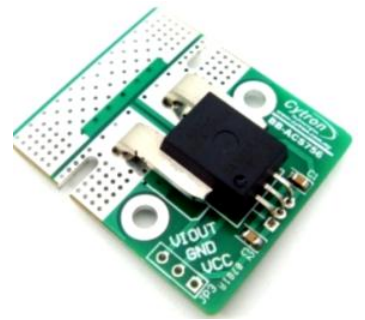

(c)

Fig. 3. (a) Arduino Uno main board, (b) XBee + IO expansion shield, and (c) 50A current sensor.

\subsection{Microcontroller programming}

The Arduino Uno is a microcontroller board based on the ATmega328. To integrate current sensor and the send the sensor data through ZigBee communication, the microcontroller needs to be programmed. Arduino microcontroller supports $\mathrm{C}++$ programming language to program it. In this project, we have used Arduino Uno board with ATmega328 microcontroller. However, before the program be started, the both ZigBee need to be given 16 bit source and destination address with its Personal Area Network (PAN) ID. PAN ID is important as it avoids conflicting from other network. One ZigBee's destination should be the other ZigBee's source address, and vice versa. For example, if we label the two Zigbee modules as ZigBee1 ZigBee2, the source and destination address of Zigbee1 is XXXX and YYYY respectively. On the other hand, the source and destination address of ZigBee2 is YYYY and XXXX respectively. The 
configuration of the two ZigBee1 and ZigBee2 for this project is shown in Table 2.

Table 2. ZigBee1 and ZigBee2 source and destination address

\begin{tabular}{|l|l|l|l|}
\hline ZigBee & Source address & Destination address & PAN ID \\
\hline ZigBee1 & 1234 & 5678 & 1111 \\
\hline ZigBee2 & 5678 & 1234 & 1111 \\
\hline
\end{tabular}

\section{Data Retrieve through LabVIEW Program}

As it is mentioned in Section 2 that the project has two ends- remote end which is connected with PV and the control unit. ZigBee enabled end is connected via serial communication with computer for data retrieval using a LabView program. The flow diagram of LabView program is shown in Fig. 4. First, it needs to open a serial connection as ZigBee is connected with the computer through USB port. Secondly, the decision making portion decides whether any incoming sensor data is received by ZigBee. If the data is available in the port, it is sent to buffer. Otherwise, the program waits for specific time and rechecks the port for next data. In this program, the incoming data is considered and read as string. Therefore, the data is converted to decimal value before it is plotted in to a graphical form or sent to a database.

In this database portion, the connectivity between LabVIEW and database is prior to be established. Once the connection is ensured, the incoming data can be fed in to the database table. The program will be terminated if the connection is failed to establish.

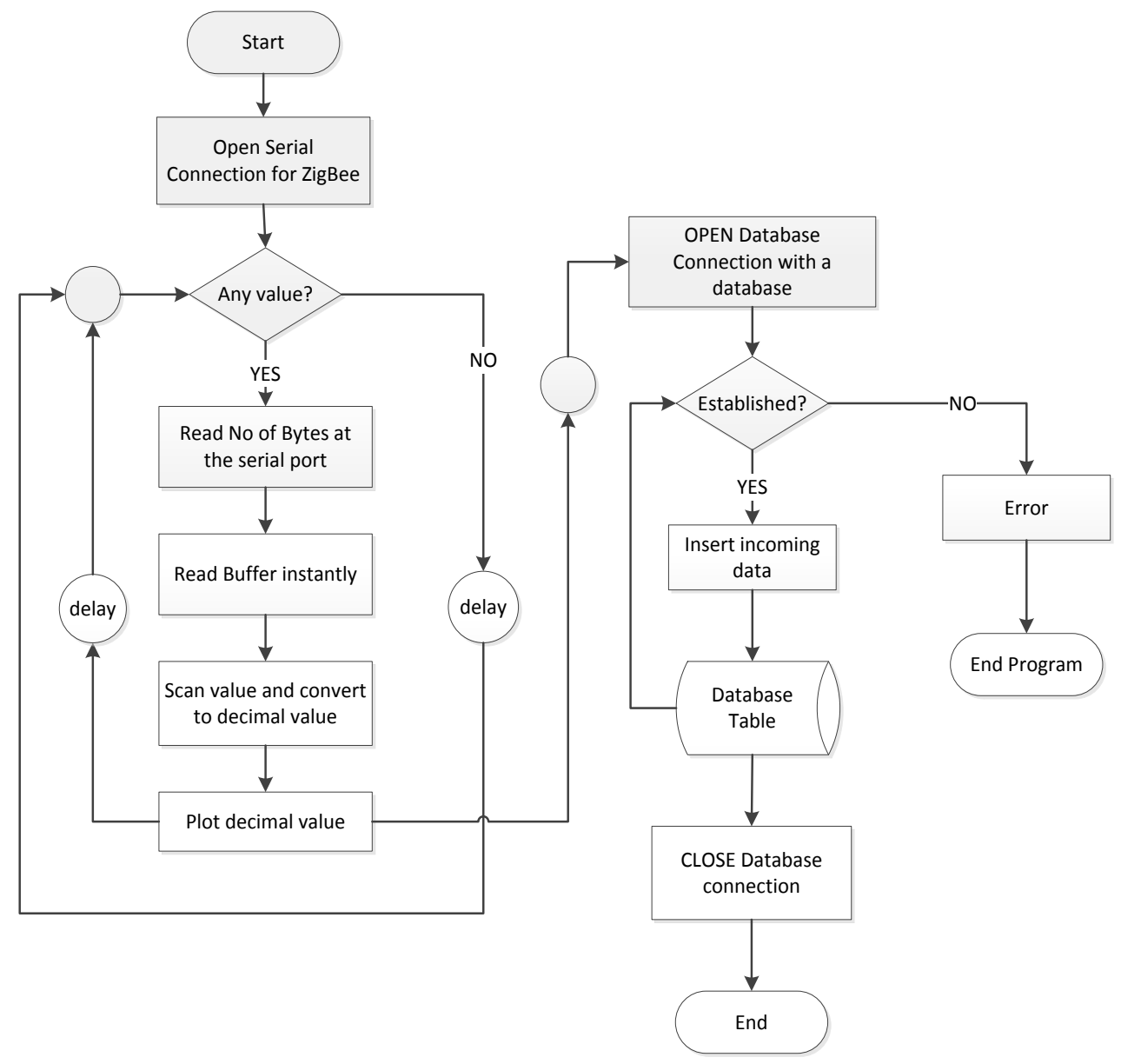

Fig. 4. Flow diagram of LabVIEW program. 


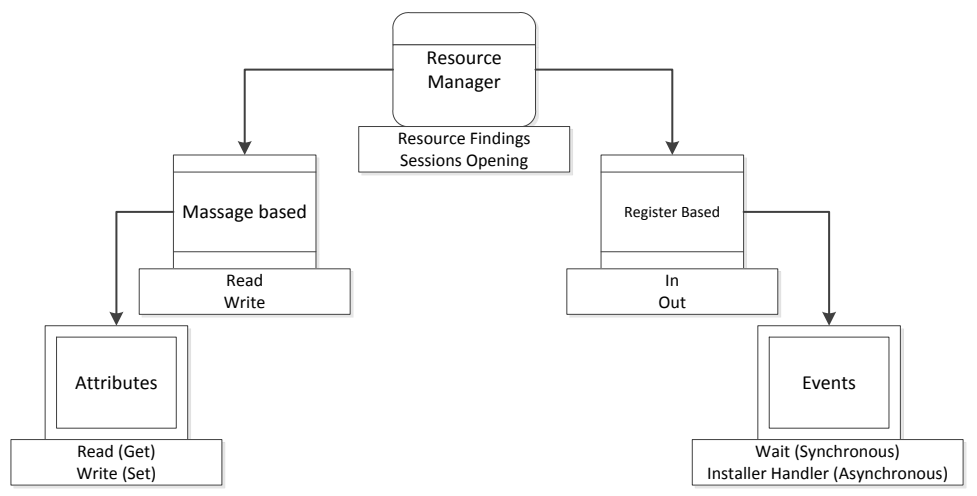

Fig. 5. Internal Structure of VISA API [11].

VISA is an object oriented language in LabView to establish connection for I/O instrumentation. It is a high-level API, but used for calling lower level drivers. VISA is capable of controlling VXI, GPIB, or Serial instruments and makes the appropriate driver calls depending on the type of instrument being used. Therefore, in this project, ZigBee connected in serial communication can be retrieved data using VISA programming module. Fig. 5 illustrates VISA architecture [11].

Fig. 6 shows the experimental works where ZigBee1 with Arduino and current sensors are installed with PV panel at distributed location. On the other hand, ZigBee2 is retrieving data through LabVIEW program placed at control center that is shown in Fig. 7. After sensing the produced PV current through current sensor, microcontroller based program is sending data through ZigBee1 to a defined address (ZigBee2). ZigBee2 is receiving measured sensor output and finally displaying in graphical form through LabVIEW program. The PV generated DC current is 1.5 A. Also Fig. 8 (a) shows generated experimental graphical data for a certain time produced from the PV panel. Fig. 8 (b) is showing the front panel view of LabView Program.

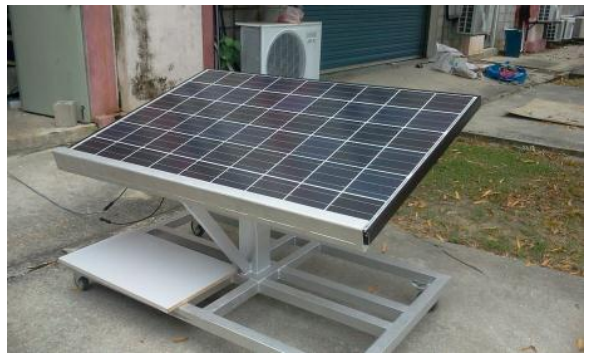

(a)

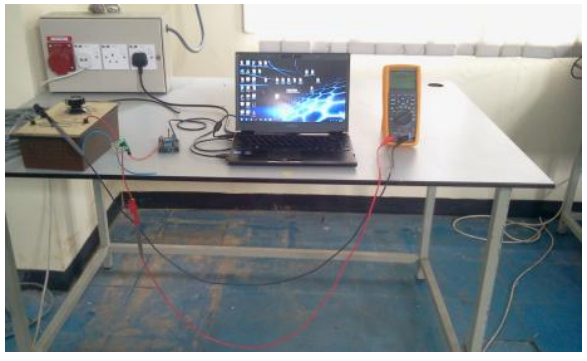

(b)

Fig. 6. Set up for the test bed of ZigBee1Sensor integrated with Arduino at remote location for measuring PV current (a) PV panel (b) ZigBee1 is connected with PV panel to retrieve PV generated current $(1.5 \mathrm{~A})$.

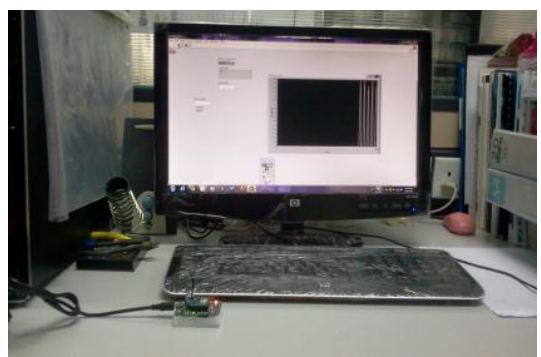

(a)

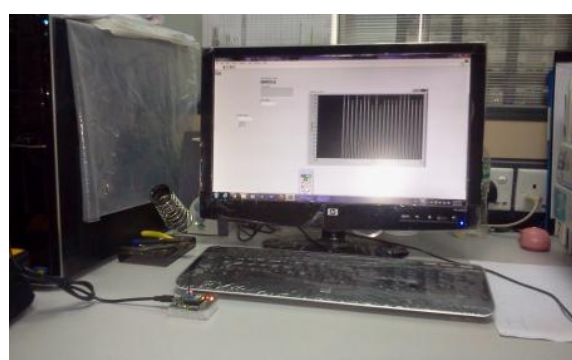

(b)

Fig. 7. Control unit, ZigBee2 location with LabVIEW Program- (a) Receiving PV generated current from ZigBee1 (b) Measured current is being displayed in real time. 


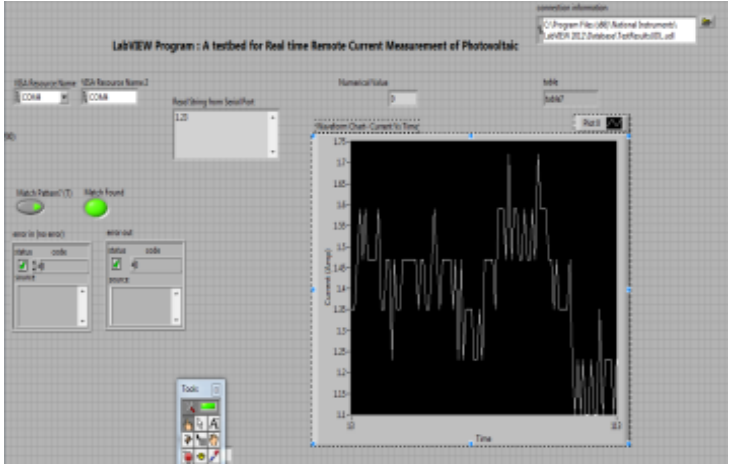

(a)

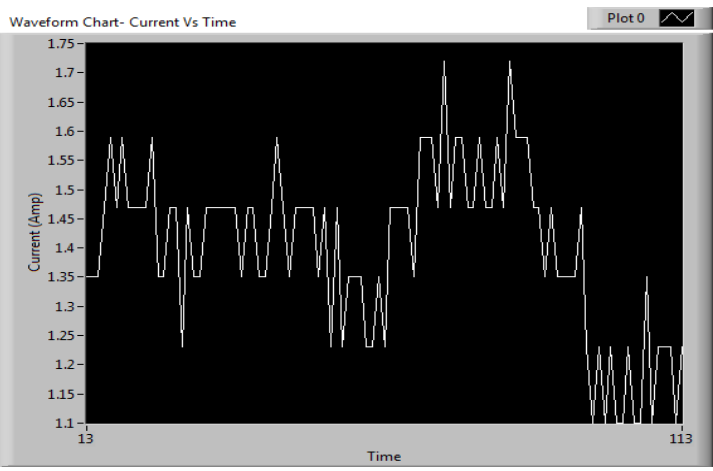

(b)

Fig. 8. (a) Front panel view of LabVIEW program (b) Measured PV produced current of a PV panel fluctuating between 1.1 A to $1.7 \mathrm{~A}$ due to PV generation factors.

\section{Conclusion}

In this project, a test bed is created for measuring the PV generated output from a distributed place. ZigBee based communication protocol is chosen for remote data sensing as it can be driven by less power. The measured real-time current output is crucial for grid control unit as the data can be used for demand management system. Moreover, this real-time data can be used to analyze to forecast demand of a certain zone which will yield minimize the energy cost. The project work can be extended to as a future work to fabricate several sensors integrated ZigBee connected with several distributed renewable generations which are situated at distance locations from each other and send those data to control unit.

\section{Acknowledgements}

The authors would like to thank Ministry of Higher Education (MOHE), Malaysia for the support by a Research Grant, No. 5450680 and Universiti Putra Malaysia (UPM) Internal Research Grant, No. 9321100RUG.

\section{References}

[1] Sun X, et al. Sensor-based high voltage electric energy meter and its application. In: Proc. of 2008 China International Conference on Electricity Distribution, 2008:1-4.

[2] Chen K-L; Tsai Y-P, et. al. Using coreless hall effect sensor for accurate current measurement in ZigBee based wireless sensor network. In: Proc. of the 25th IEEE Canadian Conference on Electrical \& Computer Engineering, 2012:1-4.

[3] Xiao C, Zhao L, et al. An overview of integratable current sensor technologies. In: Proc. of the 38th IAS Annual Meeting Conference Record of the Industry Applications, 2003:1251-1258.

[4] Cheng X, Zhang Z, Li F. Study of magnetic properties for iron core in a closed loop hall current sensor. In: Proc. of the 13th International Conference on Electronic Packaging Technology and High Density Packaging, 2012:575-578.

[5] Ajbl A, Pastre M, Kayal M. A fully integrated hall sensor microsystem for contactless current measurement. IEEE Sensors Journal, 2013; 13(6):2271-2278.

[6] Yan Y, Qian Y, Sharif H, Tipper D. A survey on smart grid communication infrastructures: motivations, requirements and challenges. IEEE Communications Surveys \& Tutorials, 2013; 15(1):5-20.

[7] Yi P; Iwayemi A, Zhou C. Developing Zigbee deployment guideline under WiFi interference for smart grid applications. IEEE Tras. on Smart Grid, 2011; 2(1):110-120.

[8] Ullo S, Vaccaro A, Velotto G. The role of pervasive and cooperative sensor networks in smart grids communication. Proc. of the 15th IEEE Mediterranean Electrotechnical Conference, 2010:443-447.

[9] Heile B. How Zigbee is being used in the smart grid. Presented at: World Leadership Forum 2011: Strong Smart Grid Week, ZigBee Alliance, 2011.

[10] Zhang Q, Sun Y, Cui Z. Application and analysis of Zigbee technology for smart grid. In: Proc. of International Conference on Computer and Information Application, 2010:171-174.

[11] National Instruments. (Jul 17, 2012). Instrument Control in LabVIEW Tutorial. [Online]. Available at: http://www.ni.com/white-paper/3511/en 